\title{
Deprivation, disease, and death in Scotland: graphical display of survival of a cohort
}

\author{
James Chalmers, Simon Capewell
}

Death rates are widely used as a convenient way of summarising important aspects of health in a population. However, they are not always easy to interpret, and even when techniques such as standardisation are used the impact of premature death may be difficult to assess. Cohort analysis provides summary information that takes account of age at death and that can be displayed graphically in a readily understood format. First used in 1870 by the statistician William Farr, this straightforward technique deserves wider use. ${ }^{1}$

It is widely accepted that deprivation increases the risk of early death. However, the age at which death from specific causes occurs and the relative contributions of these causes to mortality are rarely described clearly. ${ }^{2}$ We used data on survival in a cohort of middle aged people, divided into groups according to deprivation, to examine the relation between age, deprivation, and causes of death in a straightforward and graphical way.

\section{Methods and results}

From census projections the General Register Office for Scotland provided information on the number of people who were born in 1920 and who survived to the end of 1974. For each year from 1975 to the end of 1997 we looked at all registered deaths by specific cause and used postcodes of residence at time of death to assign Carstairs deprivation scores to each person. ${ }^{3}$ We grouped the scores into five standard groups on the basis of the distribution of deprivation scores in the total population. A disease specific survival chart was then built up for each of the deprivation groups, on the basis of two assumptions: firstly, that the census projections of people in the total population who were born in 1920 and who survived to the end of 1974 can be assumed to be equally distributed among the five deprivation groups; and secondly, that a person's Carstairs score at death represents their deprivation during the last years of their life. (Scottish studies indicate that level of deprivation changes little among people of this age group. ${ }^{4}$ )

The number of people born in 1920 who were still alive at the end of 1974 was 70365 (33 208 men (47\%), 37157 women). Over the next 23 years about half of these people died (19 912 men (28\% of the original total) and 15202 women (22\%)). Deprivation had a strong effect on mortality: $44 \%$ of men in the least deprived fifth had died by the end of 1997, compared with $72 \%$ of men in the most deprived fifth (fig 1); in women the corresponding figures were $30 \%$ and $50 \%$ (fig 2). Men in the most deprived fifth reached an equivalent mortality seven years on average earlier than men in the least deprived fifth, and the equivalent difference in women was six years.

\section{Comment}

Cohort survival graphs are a good way to show that deprived people die from the same conditions as affluent people but some years earlier overall. The risk of premature death in middle age is much greater in the most deprived fifth than in the least deprived. The proportion of deaths from conditions related to smoking, such as lung cancer and respiratory disease, is slightly greater in the most deprived fifth. In women the proportion of deaths from malignant neoplasms is greater in the least deprived fifth than in the most deprived. The graphs clearly show that no specific diseases are related to deprivation; rather, it is as though deprived people have the same mortality pattern as affluent people who are seven years older The car analogy of Graham Watt, professor of general

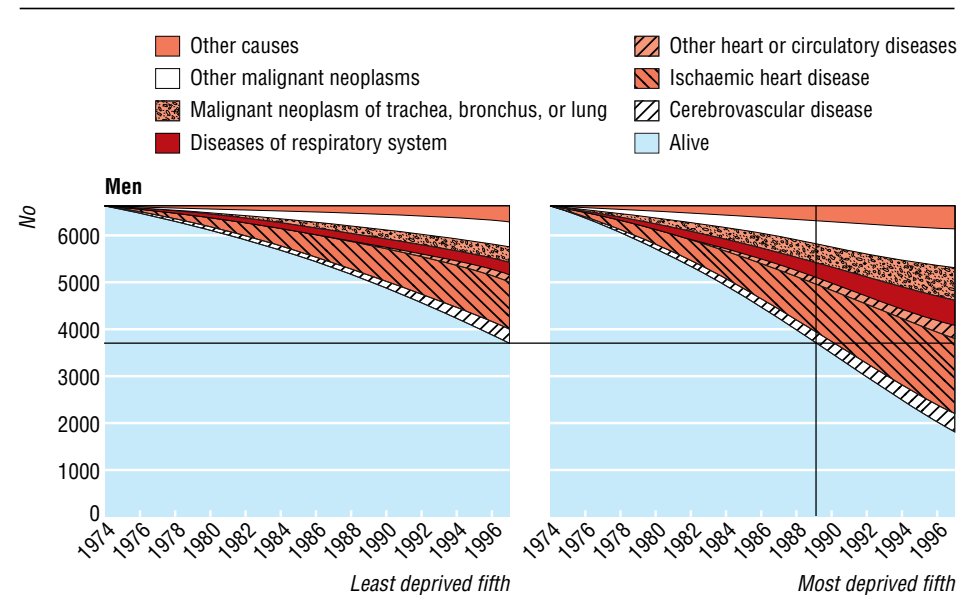

Fig 1 Survival from 1974 to end of 1997 of men born in 1920 and who were alive at end of 1974 (cumulative outcome in terms of cause of death)

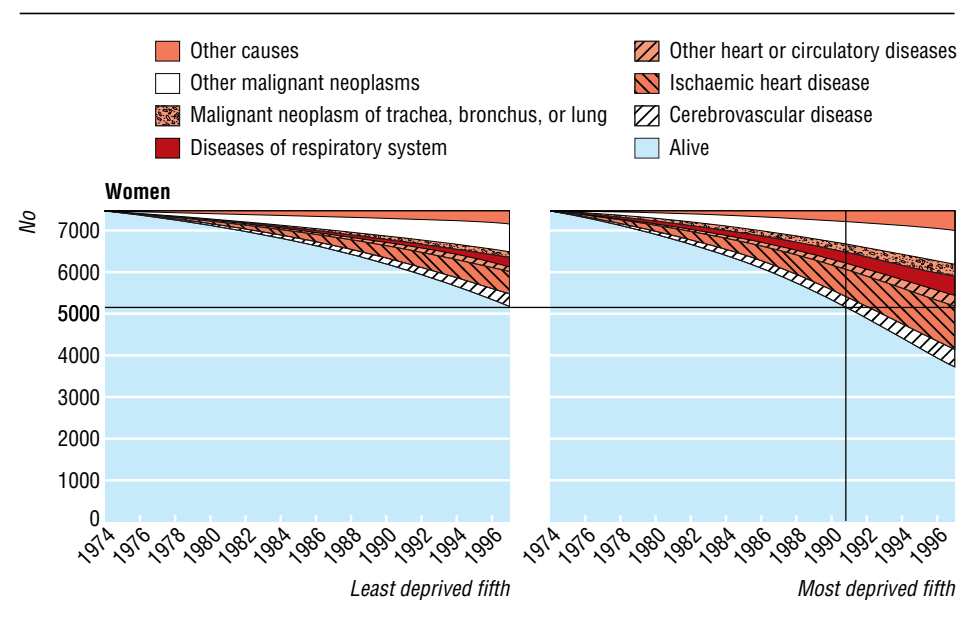

Fig 2 Survival from 1974 to end of 1997 of women born in 1920 and who were alive at end of 1974 (cumulative outcome in terms of cause of death) 
Department of Public Health, University of

Liverpool,

Liverpool L69 3GB

Simon Capewell professor of clinical epidemiology

Correspondence to: J Chalmers jim.chalmers@isd. csa.scot.nhs.uk practice at the University of Glasgow, is helpful: "More miles on the clock" (G Watt, personal communication, 1999).

The composition of the cohort changed markedly from 1974 to 1997, with a much greater proportion of survivors in 1997 being in the least deprived fifth than in the most deprived ( $27 \%$ and $14 \%$, respectively, of men and $23 \%$ and $17 \%$ of women).

These effects of deprivation on premature death may not be obvious in tables or histograms. ${ }^{2}$ Our cohort survival graphs, which can be constructed without complex links between records, clearly show the relation between deprivation and mortality.
We thank the General Register Office for providing the mortality and population data and David Murphy of the Information and Statistics Division for his help in the initial analyses.

Contributors: JC had the original idea, which he then developed with SC. JC did the analyses. Both authors drafted and wrote the paper.JC is the guarantor for the paper.

Competing interests: None declared.

1 Langmuir AD. William Farr: founder of modern concepts of surveillance. Int J Epidemiol 1976;5:13-8.

2 Loevinsohn BP. Data utilization and analytical skills among mid-level health programme managers in a developing country. Int J Epidemiol 1994;23:194-200.

3 Carstairs V, Morris R. Deprivation and health in Scotland. Aberdeen: Aberdeen University Press, 1991.

4 Hart CL, Smith GD, Blane D. Social mobility and 21 year mortality in a cohort of Scottish men. Soc Sci Med 1998;47:1121-30.

(Accepted 21 September 2001)

\section{Mobility impairments and use of preventive services in women with multiple sclerosis: observational study}

Eric Cheng, Lawrence Myers, Sheldon Wolf, Deborah Shatin, Xin-Ping Cui, George Ellison, Thomas Belin, Barbara Vickrey

Editorial by Wagner

Department of

Neurology,

University of

California,

650 Charles Young

Drive South,

Box 951736,

Los Angeles,

CA 90095-1769,

USA

Eric Cheng

health services fellow

Lawrence Myers

professor

Sheldon Wolf

physician

George Ellison

professor emeritus

Barbara Vickrey

associate professor

Center for Health

Care Policy and

Evaluation, 9000

Bren Road East,

Minnetonka

MN 55343, USA

Deborah Shatin

senior researcher

Department of

Biostatistics,

University of

California,

Los Angeles

Xin-Ping Cui

graduate student

Thomas Belin

associate professor

Correspondence to:

E M Cheng

emc@ucla.edu

BMJ 2001;323:968-9
Use of preventive health services is affected by factors such as patient demographics, clinical characteristics, type of provider, and type of healthcare system. ${ }^{1}$ Although people with multiple sclerosis may have impaired mobility, their lifespans are similar to age matched population controls. They therefore need standard preventive services to prevent early mortality. We evaluated the relation between mobility and use of preventive services in women with multiple sclerosis.

\section{Participants, methods, and results}

In 1996, we sent questionnaires to 1164 adults with multiple sclerosis who had received outpatient care in 1993 or 1994 from one of three systems of health care (two forms of managed care and fee for service insurance) in two regions of the United States. ${ }^{2}$ The overall response rate was $80 \%(930 / 1164)$. We report here survey analyses from the 713 women respondents.

We collected self reported rates of cervical smear testing, mammography, and breast examination (if over age 50), blood pressure checks, cholesterol screening, and physician assessment of health habits. We assessed these rates according to the patient's mobility level (fully ambulatory, ambulatory with help, and not ambulatory) and compared them with Healthy People 2000 recommendations. ${ }^{3}$ For each preventive service, we used logistic regression to model the relation between that service, mobility, patient demographics, comorbidity, ${ }^{2}$ system of health care, indicators for having a primary care physician and a multiple sclerosis physician, and specialty of these physicians.

The mean age of the women was 47 years; $86 \%$ were white and $40 \%$ had a four year college degree. Overall rates for cervical smear tests, breast examinations, and mammography exceeded Healthy People 2000 recommendations, but rates were highest for the ambulatory group and lowest for the non-ambulatory group $(\mathrm{P} \leqslant 0.05$, table). Cervical smear testing was below Healthy People 2000 goals for the ambulatory with help and non-ambulatory groups. In contrast, rates for general preventive services did not differ by mobility.

In the multivariable models, ambulatory patients had 5.32 times the odds of having a cervical smear test, 3.62 times the odds of having a breast examination, and 3.24 times the odds of having mammography relative to non-ambulatory patients (all $\mathrm{P}<0.05$ ). Older age was associated with a lower rate of cervical smear tests; however, no other variables were related to receipt of women's preventive services. Except for an increased odds of assessing eating habits in the non-ambulatory group, mobility status did not affect the odds of receiving general preventive services.

\section{Comment}

Use of women's preventive health services was lower in non-ambulatory women than in fully or partially ambulatory women with multiple sclerosis. These results are the same as those in a previous population based US study of women with and without mobility impairments due to various conditions, ${ }^{4}$ even though women in our study had a single chronic condition, were younger, were more educated, and all had health insurance and a regular source of care in health systems that met broad national screening goals.

There are several possible explanations for these findings. Doctors may believe that such patients do not have an adequate life expectancy to warrant women's preventive screening. However, such attitudes would be incompatible with the high rates of blood pressure and cholesterol checks. Alternatively, patients may be reluctant to undergo screening services that are potentially uncomfortable or embarrassing. ${ }^{5}$ A third possibility is that the medical systems cannot easily accommodate patients with mobility impairments, who may require access to specialised equipment and extra time. 